\title{
ESS
}

\section{Electrochemical Flow-Cell Setup for In Situ X-ray Investigations}

\section{Cell for SAXS and XAS at Synchrotron Facilities}

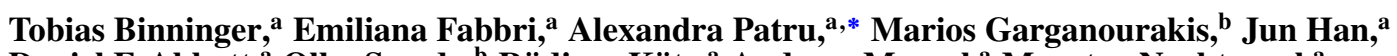 \\ Daniel F. Abbott, ${ }^{\text {a }}$ Olha Sereda, ${ }^{\mathrm{b}}$ Rüdiger Kötz, ${ }^{\text {a }}$ Andreas Menzel, ${ }^{\mathrm{a}}$ Maarten Nachtegaal, ${ }^{\mathrm{a}}$ \\ and Thomas J. Schmidt ${ }^{\mathrm{a}, \mathrm{c}, *, \mathrm{z}}$
}

\author{
${ }^{a}$ Paul Scherrer Institut, CH-5232 Villigen PSI, Switzerland \\ ${ }^{b}$ Centre Suisse d'Electronique et de Microtechnique SA, CH-2002 Neuchâtel, Switzerland \\ ${ }^{c}$ ETH Zürich, Laboratory of Physical Chemistry, CH-8093 Zürich, Switzerland
}

\begin{abstract}
An electrochemical three-electrode flow-cell is presented for in situ small-angle X-ray scattering (SAXS) and X-ray absorption spectroscopy (XAS) experiments in transmission mode at synchrotron X-ray sources. The cell also allows for in situ XAS performed in fluorescence mode. Constant experimental conditions, even under moderate gas evolution, are provided by the electrolyte flow with controlled gas saturation. A special configuration of working and counter electrode, respectively, yields low residual ohmic resistance in three-electrode measurements that enables the study of thick porous electrodes of active high surface area materials. The cell proved its functionality and reliability in two studies: First, an in situ anomalous SAXS experiment for the high-potential degradation properties of a $\mathrm{Pt} / \mathrm{IrO}_{2}-\mathrm{TiO}_{2}$ catalyst for the oxygen reduction reaction at polymer electrolyte fuel cell cathodes; and second, an in situ XAS study of the electronic state of $\mathrm{Ir}$ centers inside an $\mathrm{IrO}_{2}-\mathrm{TiO}_{2}$ catalyst under oxygen evolution conditions.

(C) The Author(s) 2016. Published by ECS. This is an open access article distributed under the terms of the Creative Commons Attribution 4.0 License (CC BY, http://creativecommons.org/licenses/by/4.0/), which permits unrestricted reuse of the work in any medium, provided the original work is properly cited. [DOI: 10.1149/2.0201610jes] All rights reserved.
\end{abstract}

Manuscript submitted June 15, 2016; revised manuscript received July 26, 2016. Published August 5, 2016.

Modern research in electrocatalysis makes extensive use of in situ X-ray techniques that provide information about the structure and the electronic state of catalyst materials under electrochemical potential control. The reason for this is the limited, merely indirect information about the state of the catalyst that can be deduced from purely electrochemical testing like cyclic voltammetry $(\mathrm{CV})$ which often does not allow for an unambiguous interpretation of the data. In order to develop an understanding at a more fundamental level, additional information is required about the potential-dependent state of electrocatalyst materials that can be provided by synchrotron-based techniques like X-ray scattering or X-ray absorption spectroscopy.

One example is the investigation of polymer electrolyte fuel cell (PEFC) Pt cathode catalyst degradation. Different mechanisms have been proposed for the loss of electrochemically active Pt surface area (ECSA) that occurs most severely at transient high-potential spikes during PEFC start and stop. ${ }^{1,2}$ Processes like agglomeration of primary $\mathrm{Pt}$ particles due to migration or carbon support corrosion, $\mathrm{Pt}$ loss due to dissolution, and growth of primary $\mathrm{Pt}$ nanoparticles due to dissolution/redeposition cycles have been considered ${ }^{3,4}$ and quantified for different operation conditions and electrochemical environments. The most common technique applied for this purpose is transmission electron microscopy (TEM), which has the convenient advantage that changes of the Pt nanoparticle structure can be directly visualized, especially with the use of identical location TEM (IL-TEM). ${ }^{5}$ Although successfully demonstrated, ${ }^{6}$ in situ TEM remains limited to certain electrochemical systems. Whereas the strength of TEM lies in the direct imaging of individual catalyst particles, it is challenging to extract quantitative statistical information about the entire catalyst sample from TEM analysis. Finally, the distinction of Pt nanoparticles from the support material phase becomes very difficult if the latter contains heavy elements, e.g. if a metal oxide is used as support. Figure 1 compares a TEM image of a typical $\mathrm{Pt} / \mathrm{C}$ catalyst with the one of a $\mathrm{Pt} / \mathrm{IrO}_{2}-\mathrm{TiO}_{2}$ catalyst. Whereas the carbon-supported $\mathrm{Pt}$ nanoparticles are clearly visible, no Pt particles can be distinguished on the Ir-containing support oxide.

As an alternative, small-angle X-ray scattering (SAXS) provides structural information at the nanoscale in reciprocal space and can be applied in situ during electrochemical testing. ${ }^{7}$ In addition, SAXS scattering curves intrinsically contain structural information statistically averaged over millions of Pt nanoparticles, so that a direct quan-

*Electrochemical Society Active Member.

${ }^{\mathrm{z} E}$-mail: thomasjustus.schmidt@psi.ch titative analysis of the entire catalyst sample is provided. Finally, the tunability of the X-ray photon energy at synchrotron sources enables the use of anomalous SAXS (ASAXS) in order to extract elementspecific structural information. ${ }^{8}$ The energy-dependent variation of the X-ray scattering cross section of Pt close to the Pt- $\mathrm{L}_{\mathrm{III}}$ absorption edge can be used to separate the Pt nanoparticle scattering from the scattering of the support material ${ }^{9}$ even for the strongly scattering $\mathrm{IrO}_{2}-\mathrm{TiO}_{2}$ support material of the catalyst presented in Figure $1 \mathrm{~b}$.

A second example of synchrotron-based in situ techniques for electrocatalysis is the use of X-ray absorption spectroscopy (XAS) in the research on oxygen evolution reaction (OER) catalysts. In situ-acquired XAS spectra contain information about the potentialdependent oxidation state (X-ray absorption near edge structure, XANES) and the potential-dependent local coordination structure (extended X-ray absorption fine structure, EXAFS) of specific elements in metal oxide OER catalysts. Such information is crucial in order to understand the catalytically active state and the degradation properties of the metal oxide catalyst under OER conditions. ${ }^{10-13}$

Electrochemical cells for in situ X-ray spectroscopy studies of electrocatalysts have been published based on a PEFC-type design with a non-liquid polymer electrolyte. ${ }^{14-17}$ Although for PEFC-related catalyst investigations such setups are advantageous, their use remains restricted to available polymer electrolytes. Especially for studies in alkaline environment, a cell design for liquid electrolytes is desirable. Furthermore, the provision of constant experimental conditions

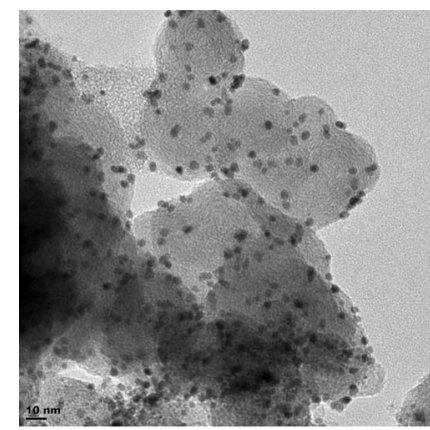

(a)

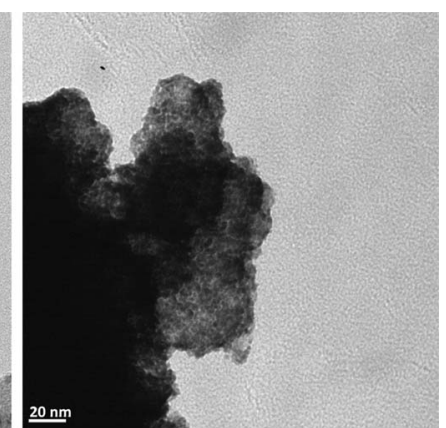

(b)
Figure 1. TEM images of a $20 \mathrm{wt} . \% \mathrm{Pt} / \mathrm{C}$ (Vulcan XC-72R) catalyst (a) and an 8.8 wt. $\% \mathrm{Pt} / \mathrm{IrO}_{2}-\mathrm{TiO}_{2}$ catalyst (b). Image (a) was acquired on a Tecnai $\mathrm{G}^{2}$ and (b) on a Phillips CM200 electron microscope, respectively, both operated at $200 \mathrm{kV}$. 
in PEFC-type in situ cells during long-term investigations is questionable in light of the possibility of X-ray induced polymer electrolyte degradation. $^{18}$

Few in situ cell designs for liquid electrolytes have been presented, each with specific advantages and disadvantages. The cell presented in Ref. 19 worked with liquid electrolyte soaked in filter paper which renders it not applicable for the study of gas evolution reactions like OER. The three-electrode in situ cell described in Ref. 20 has an open electrolyte chamber that does not allow for electrolyte flow. Although in Ref. 7 an in situ cell is shown where the electrolyte was flowed through a window compartment, sketch and corresponding description of the flow-cell design remain very vague which renders reproduction extremely difficult.

In this publication (Part I.), we present in detail the design of an electrochemical three-electrode flow-cell for in situ SAXS and XAS experiments in liquid electrolytes at synchrotron X-ray sources. The cell was specifically designed for the study of technically relevant high surface area catalyst materials in porous electrode layers with a similar morphology as in technical applications. The main advantage of the cell in comparison to other three-electrode in situ cell designs can be seen in the liquid electrolyte flow with controlled gas saturation. In this way, X-ray induced degradation and heating of the electrolyte is prevented and highly controlled electrochemical conditions are provided even during long-term catalyst degradation experiments. The liquid electrolyte environment enables complete electrochemical utilization of the studied catalyst layer. Furthermore, the cell allows for in situ studies of OER catalysts where electrolyte flow is crucial in order to carry away evolved gases and prevent gas filling of the cell. In addition, the special opposite arrangement of working and counter electrode, respectively, results in a low ohmic resistance in threeelectrode measurements, which guarantees high-quality performance even for large electrochemical currents produced by thick active material layers. The latter are required for a high signal-to-noise ratio in $\mathrm{X}$-ray experiments with short acquisition times. Finally, the complete tightness of the cell assures reliable experiments without electrolyte leakage-related danger for other equipment.

The flow-cell was applied and proved reliable in two in situ experiments: First, an in situ SAXS study of the PEFC cathode-relevant degradation properties of the $\mathrm{Pt} / \mathrm{IrO}_{2}-\mathrm{TiO}_{2}$ catalyst presented in Figure $1 \mathrm{~b}$; and second, an in situ XAS study of the electronic state of an $\mathrm{IrO}_{2}-\mathrm{TiO}_{2}$ metal oxide catalyst under OER conditions.

In a second publication (Part II.), ${ }^{21}$ a second flow-cell for in situ SAXS experiments on a multi-purpose laboratory X-ray diffractometer is presented, which is based on the same design principles. However this flow-cell has a miniature geometry that fits into the sample holder of the laboratory diffractometer. Due to the fixed X-ray energy of such systems, anomalous SAXS cannot be used for the extraction of the net Pt signal. Details of SAXS data analysis and background scattering subtraction for this case are discussed in the same publication.

\section{Experimental}

Electrochemical flow-cell for in situ SAXS/XAS.-Since both SAXS and XAS are conveniently performed in transmission mode, a common flow-cell design for both techniques was possible. Special emphasis was given to achieve good X-ray transmission through an electrolyte layer of $2 \mathrm{~mm}$ thickness and maintain a low ohmic resistance and homogeneous current distribution on the active material layer at the same time. Furthermore, the design was optimized for the study of high surface area catalyst materials in porous electrode layers with good control of electrode morphology.

PEEK was chosen as material of the cell because of its chemical resistance towards diluted acidic and alkaline electrolytes and its good mechanical stability and processability. The cell consisted of three parts, which are presented in Figure 2, and which are referred to as 'bottom part', 'middle part', and 'top part' in the following. Since the X-ray beam at a synchrotron beamline generally propagates horizontally, the cell was placed vertically as shown in Figure 3 with the top part facing towards the incoming beam. The main electrochem- ical compartment was formed by the central hole in the middle part which was confined by two Kapton foils, one placed in between the middle part and top part, and one placed in between the middle part and bottom part, which served as windows for the X-ray beam. The special Kapton foils (DuPont Kapton 200RS100, $50 \mu \mathrm{m}$ thickness) were electrically conductive on one side which faced inwards into the electrochemical compartment. In this way, the bottom Kapton foil could be used as substrate for the working electrode and the top Kapton foil as counter electrode. The studied material was deposited by spray-coating (cf. Experimental section) on the bottom Kapton foil. In this configuration, the X-ray beam was first attenuated by the 2 $\mathrm{mm}$ thick electrolyte layer defined by the thickness of the middle part inner region before reaching the active material layer so that possible beam-induced degradation effects of the studied material were minimized. The central electrochemical compartment was tightened by two O-rings, placed in grooves of the top part and the bottom part, that pressed the Kapton foils against the middle part planes. The electrolyte flowed through a channel of $1 \mathrm{~mm}$ diameter that was precisely drilled into the middle part and which was connected to external tubing by standard tube fittings via two threaded holes at the side ends of the channels. A second electrolyte compartment, which was placed upstream of the main central compartment along the electrolyte channel, allowed to insert a $\mathrm{Ag} \mid \mathrm{AgCl}$ reference electrode (Harvard Apparatus Low-Leakage with $\mathrm{NaCl}$ filling solution and $2 \mathrm{~mm}$ diameter) into the electrolyte stream via a threaded hole in the top part with the use of 2 $\mathrm{mm}$ tube fittings (cf. Figure 3). This arrangement with the reference electrode placed upstream avoided electrolyte contact loss between the three electrodes in the case of gas evolution. Gas bubbles formed either on the working or on the counter electrode, respectively, were carried away downstream which was most important for investigations under oxygen evolution conditions. Electrolyte flow was controlled by a syringe pump which, due to the tightness of the cell, could be used in withdrawal mode and placed downstream. In this way, inexpensive plastic syringes could be used to collect the waste electrolyte and the fresh electrolyte was drawn via tubing from a reservoir in a clean glass bottle that was purged with $\mathrm{N}_{2}$ in order to de-aerate the electrolyte.

The conductive Kapton electrodes were contacted via gold-coated spring contacts that were pressed into in-house developed PEEK screws (cf. Figure 4a). These contacts were screwed into respective holes in the top part and the bottom part in order to contact the bottom working electrode via the screw contacts in the top part, and the top counter electrode via the screw contacts in the bottom part, respectively. Precisely located holes in the middle part allowed the spring contacts to pass through. Both conductive Kapton electrode substrates had an individual shape in order to let the spring contacts for the respective opposite electrode pass by (cf. Figures 4b, 4c). Marking lines were engraved into the top part and the bottom part for a precise positioning of both Kapton electrodes.

In order to improve the limited sheet resistance of the conductive Kapton surface, a gold layer of 50-100 nm thickness was sputtered onto the conductive Kapton foils. A circular central region was masked during the $\mathrm{Au}$-sputtering process in order to provide a $\mathrm{Au}$-free window for the X-ray beam to pass through without parasitic scattering or absorption from the Au layers. Porous electrode layers (thickness 10-50 $\mu \mathrm{m})$ of the studied high surface area catalyst materials were prepared by spray-coating on the working electrode conductive Kapton foil (cf. Experimental section). In-house designed masks for the spraying process guaranteed a well-defined circular shape of the porous electrode layers precisely located on the Au-free central region of the Kapton substrate (cf. Figure 4b). The conductive layer of the Kapton foil and the sputtered $\mathrm{Au}$ layer provided electrical connection between the porous material layer at the center of the Kapton substrate and the contact points of the electrical spring contacts. In order to improve the counter electrode capacity, two circular shaped layers of high surface area carbon were deposited on the counter electrode Kapton foil. These two circular counter electrode layers were sprayed with the use of masks on both sides of the circular Au-free region (cf. Figure 4c). In this way, the X-ray beam did not pass through the porous carbon 
(a)

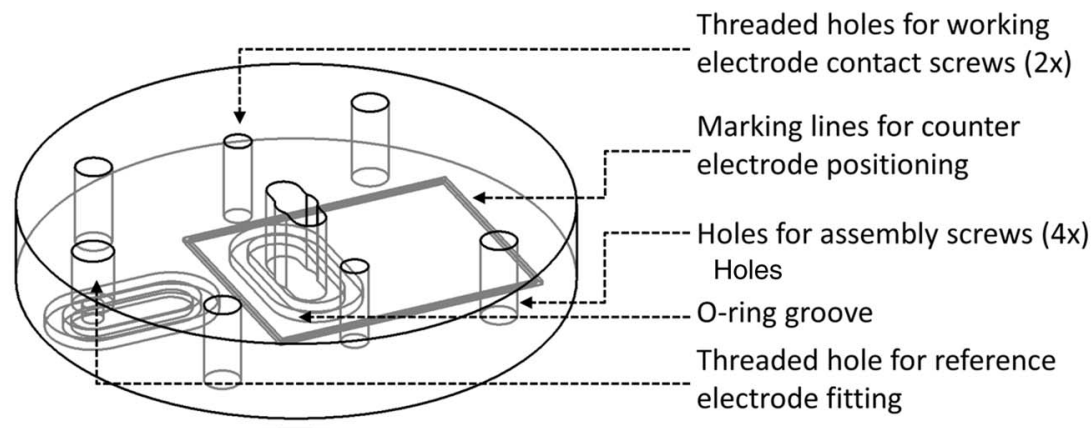

(b)

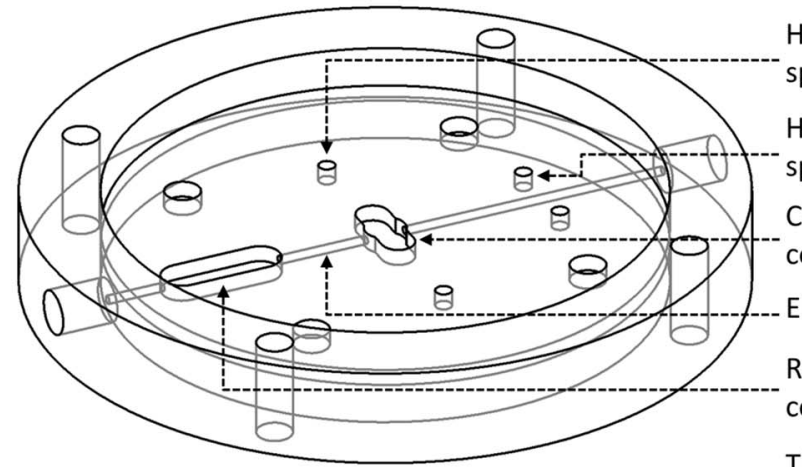

Holes for working electrode spring contacts $(2 \mathrm{x})$

Holes for counter electrode

spring contacts $(2 \mathrm{x})$

Central electrochemical

compartment

Electrolyte channel

Reference electrode

compartment

Threaded holes for counter electrode contact screws $(2 x)$

(c)

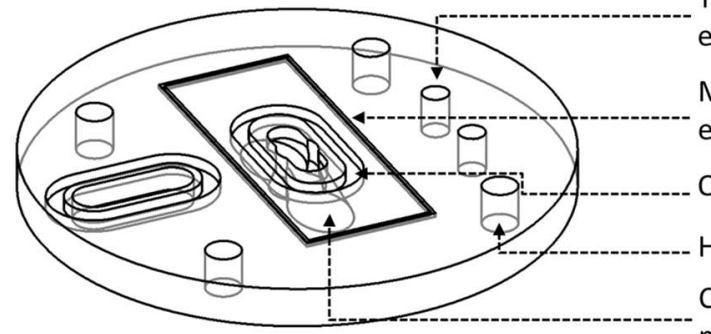

Marking lines for working

electrode positioning

O-ring groove

Holes for assembly screws $(4 \mathrm{x})$

Conical drillings for fluorescence mode XAS

Figure 2. Views of the flow-cell top part (a), middle part (b), and bottom part (c).
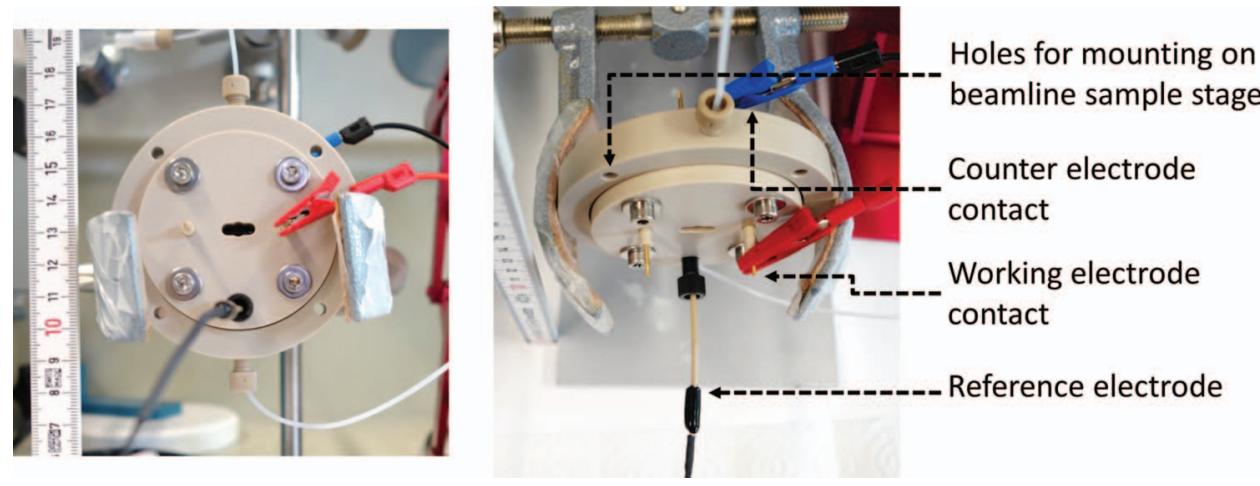

Figure 3. Pictures of the flow-cell in electrochemical three-electrode operation. Scale in $\mathrm{cm}$.

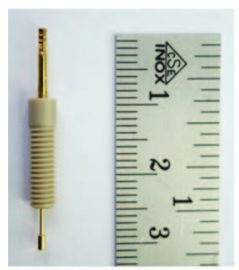

(a)

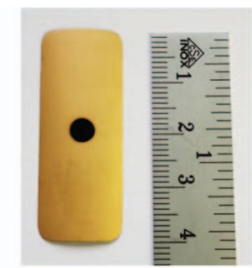

(b)

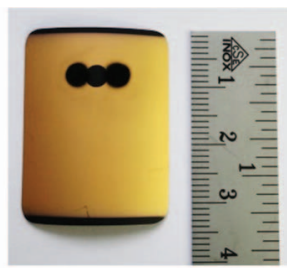

(c)

Figure 4. Spring contact pressed into PEEK screw (a), working electrode (b), and counter electrode (c). Left scale in $\mathrm{cm}$.

layers, thus avoiding parasitic scattering thereof. The special shape of the central hole in the middle part guaranteed that the two counter electrode layers were fully accommodated within the electrolyte-filled compartment.

In addition to SAXS and XAS experiments in transmission geometry, the cell was designed to also allow for XAS experiments in fluorescence mode. For this purpose, two conical holes were drilled in the bottom part at $45^{\circ}$ angles with respect to the plane. Both holes directed towards the active material layer, one for the incoming X-ray beam, and the other one for the emitted fluorescence light to reach the detector. 
In situ anomalous SAXS experiments.-In situ anomalous SAXS experiments were performed to study PEFC-relevant degradation properties of an $8.8 \mathrm{wt} . \% \mathrm{Pt} /\left(\mathrm{IrO}_{2}\right)_{0.66}-\left(\mathrm{TiO}_{2}\right)_{0.34}$ catalyst provided by Umicore AG \& Co KG. This material served as benchmark for the research on durable PEFC cathode Pt catalysts with conductive metal oxide support for the oxygen reduction reaction (ORR). ${ }^{22,23}$ Electrodes were prepared by spray-coating onto the conductive Kapton substrate in a similar way as for PEFC electrodes described in Ref. 23, but with a higher Nafion ionomer content of $30 \mathrm{wt} . \%$.

Anomalous SAXS experiments were conducted at the cSAXS beamline X12SA of the Swiss Light Source (SLS, Paul Scherrer Institut, Villigen, Switzerland). SAXS patterns were recorded with a Pilatus $2 \mathrm{M}$ detector, ${ }^{24}$ which was placed at the end of a $2.1 \mathrm{~m}$ long evacuated flight tube. Scattering curves were corrected for absorption and isotropic background scattering and transformed to absolute differential scattering cross sections per sample area with the use of a calibrated glassy carbon standard. ${ }^{25}$ The net Pt scattering was extracted from the overall SAXS signal with the use of anomalous SAXS. As described in detail in Ref. 9, SAXS curves at four different X-ray energies close to the Pt- $\mathrm{L}_{\mathrm{III}}$ absorption edge were used in the analysis, and the resulting net Pt signal was fitted with the standard model of spherical Pt particles with log-normal size distribution in combination with the model for the Pt particle-support material scattering interference presented in the same reference.

The electrochemical protocol in the in situ experiment simulated the extremely corrosive conditions at PEFC cathodes during start and stop events, when the PEFC cathode potential can reach very high transient values equivalent to $1.4-1.5 \mathrm{~V}$ vs. RHE (reversible hydrogen electrode). ${ }^{1}$ For this purpose, 500 cyclic voltammetry (CV) cycles between 0.5 and $1.5 \mathrm{~V}_{\mathrm{RHE}}$ at a sweep rate of $50 \mathrm{mV} / \mathrm{s}$ were performed on the active material, and SAXS curves were recorded at the beginning, at the end, and at different intermediate stages. During SAXS acquisition, the electrode potential was kept constant at 0.5 $\mathrm{V}_{\mathrm{RHE}}$ in order to keep the Pt nanoparticles in a well-defined, reduced state. The electrolyte was $\mathrm{N}_{2}$-saturated $0.1 \mathrm{M} \mathrm{HClO}_{4}$ (prepared from Merck KGaA Suprapur $70 \% \mathrm{HClO}_{4}$ ).

Electrochemically active Pt surface areas (ECSAs) were not determined during in situ experiments. Instead, ECSA values at different stages of the same degradation protocol were determined for the same catalyst in a standard three-electrode glass cell using a thin porous catalyst layer deposited on a glassy carbon disk as described in Ref. 26. Cyclic voltammetry (CV) was performed in order to determine the ECSA from hydrogen underpotential deposition $\left(\mathrm{H}_{\mathrm{upd}}\right)$ analysis in combination with a baseline recorded in CO-saturated electrolyte as presented in Ref. 27.

In situ XAS experiments.-In situ XAS experiments were performed to study the potential-dependent oxidation state of the active iridium component in a $\left(\mathrm{IrO}_{2}\right)_{0.66}-\left(\mathrm{TiO}_{2}\right)_{0.34}$ mixed oxide catalyst (provided by Umicore AG \& Co KG) under OER conditions. The oxide was prepared by a chemical synthesis route involving a hightemperature calcination step. Working electrodes were prepared by spraying a catalyst ink suspension made of $\mathrm{IrO}_{2}-\mathrm{TiO}_{2}$, Milli-Q $\mathrm{H}_{2} \mathrm{O}$, isopropyl alcohol, and $5 \mathrm{wt}$ \% Nafion ionomer solution, onto the conductive Kapton substrate to yield a catalyst loading of approximately $0.5 \mathrm{mg}$.

X-ray absorption spectra were collected at the SuperXAS beamline of the Swiss Light Source (SLS, Paul Scherrer Institut, Villigen, Switzerland). The incident beam was collimated by a Rh-coated mirror at $2.5 \mathrm{mrad}$ and monochromatized using a channel-cut Si (111) crystal monochromator. The beam was focused with a Rh-coated toroidal mirror (at $2.5 \mathrm{mrad}$ ) down to $100 \times 100 \mu^{2}$. X-ray energy calibration was done with a Pt reference foil to the Pt- $\mathrm{L}_{\mathrm{III}}$ edge position at 11564 $\mathrm{eV}$. Ion chambers filled with $\mathrm{N}_{2}-\mathrm{Ar}$ gas mixtures were used for XAS detection in transmission mode, where the sample cell was mounted between the first and second ion chamber.

Electrochemical measurements were conducted in $0.1 \mathrm{M} \mathrm{HClO}_{4}$ electrolyte at a flow rate of $0.1 \mathrm{ml} / \mathrm{s}$. The working electrode potential was varied between 1.2 and $1.52 \mathrm{~V}_{\mathrm{RHE}}$ with potential steps of 0.1 and
$0.02 \mathrm{~V}$ in the range of 1.2-1.4 $\mathrm{V}_{\mathrm{RHE}}$ and 1.4-1.52 $\mathrm{V}_{\mathrm{RHE}}$, respectively, with a holding time of $2 \mathrm{~min}$ at each potential. The catalyst electronic structure (Ir- $\mathrm{L}_{\text {III }}$ edge) as a function of the applied potential was followed in situ by time-resolved XAS measurements (Quick-XAS). ${ }^{28}$ At each potential, after a stabilization time of $1 \mathrm{~min}, 120$ XAS spectra were collected within $1 \mathrm{~min}$ (500 ms for each XAS spectrum). These 120 spectra were then averaged to yield one effective XAS spectrum for each potential.

The Athena module of the Demeter software suite ${ }^{29}$ was used for XAS data background subtraction and edge step normalization. The analysis of the EXAFS data was performed using the Artemis module. The photoelectron wave vector for the Fourier Transform of the spectra was kept within the range of $k=3-12 \AA^{-1}$ and a $k$-weighting factor of 2 was applied during the refinement of the EXAFS data. An amplitude reduction factor of $S_{0}^{2}=0.77$ was calculated using FEFF code ${ }^{29}$ from the iridium scattering paths of bulk $\mathrm{IrO}_{2}$ while assuming the theoretical coordination numbers of the bulk oxide. The determined $S_{0}^{2}$ value was then applied in the fitting of $\mathrm{IrO}_{2}-\mathrm{TiO}_{2}$ while allowing the coordination number, bond distance, Debye-Waller factor, and energy parameters to vary.

\section{Results}

In situ anomalous $\mathrm{SAXS}$ results: $\mathrm{Pt} / \mathrm{IrO} \mathrm{O}_{2}-\mathrm{TiO}_{2}$ degradation.- - In situ SAXS curves of the bare $\mathrm{IrO}_{2}-\mathrm{TiO}_{2}$ support material without $\mathrm{Pt}$ nanoparticles are shown in Figure 5 at an early stage and at the end of the degradation protocol. No changes are visible in the scattering curves of the support, which proves the morphological stability of the latter under high potential cycling conditions.

Figure 6a shows the net Pt scattering curves at different stages of the electrochemical degradation protocol for the $\mathrm{Pt} / \mathrm{IrO} \mathrm{O}_{2}-\mathrm{TiO}_{2}$ catalyst. The scattering contribution of the $\mathrm{IrO}_{2}-\mathrm{TiO}_{2}$ support has been subtracted by ASAXS analysis, cf. Experimental section. However, as shown in Ref., 9 the particle-support interference is still present in the net $\mathrm{Pt}$ scattering curve after ASAXS analysis, which is responsible for the reduction of scattering intensity at intermediate $q$-values evident in Figure 6a, where $q$ is the magnitude of the scattering vector. This depression is most prominent in the initial Pt scattering curve, where it leads to negative values in the $q$-range between $0.4-0.6 \mathrm{~nm}^{-1}$. Pt nanoparticle growth can be established from the shift of the bump-like feature towards lower values of $q$ and larger scattering intensity. At the same time, the depression of scattering intensity resulting from the particle-support interference effect gets weaker because of the decreasing difference between support particle size and Pt particle size. ${ }^{9}$ The growth of Pt nanoparticles in the course of high-potential

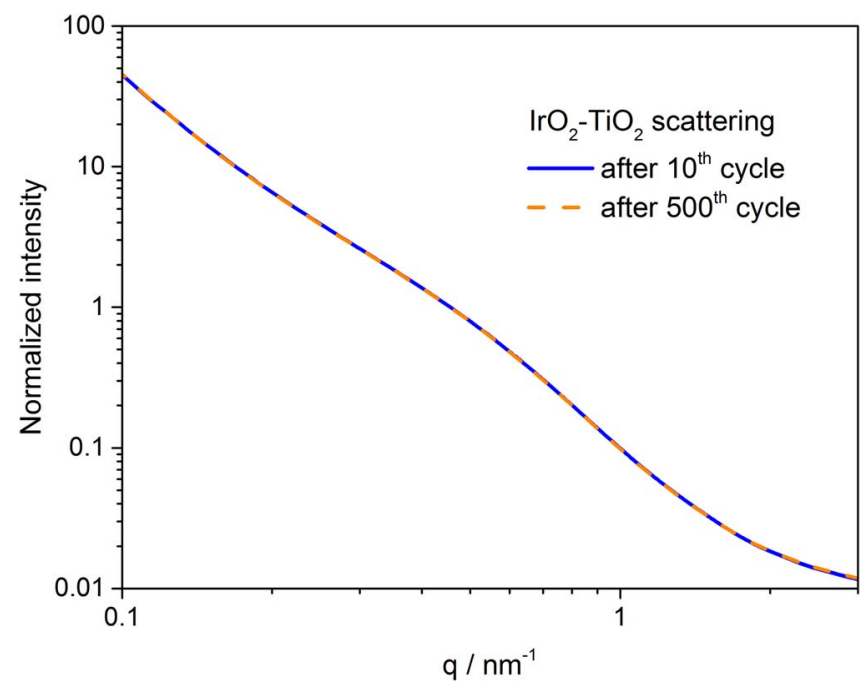

Figure 5. In situ SAXS curves of the bare $\mathrm{IrO}_{2}-\mathrm{TiO}_{2}$ support material at an early stage and at the end of the degradation protocol. 

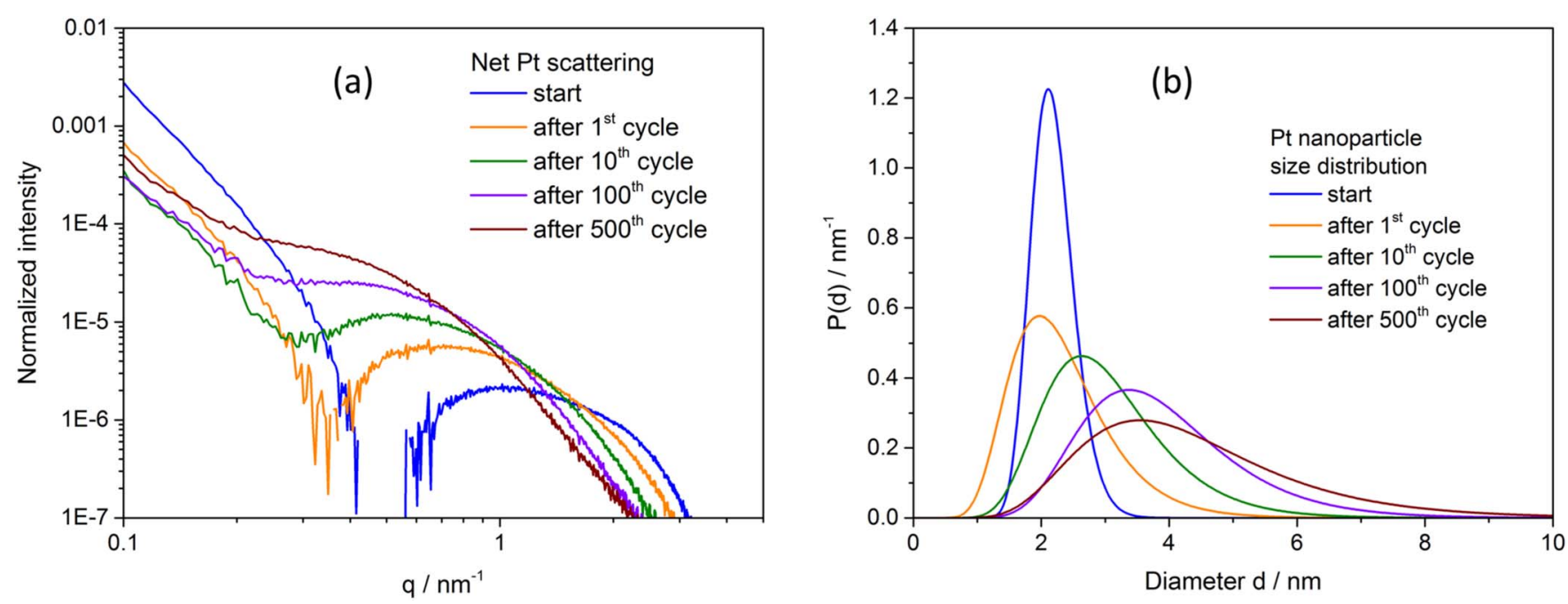

Figure 6. Net Pt scattering curves extracted by ASAXS analysis from the overall scattering signal at different stages of the electrochemical degradation protocol (a). Pt nanoparticle size distributions resulting from fitting the Pt scattering curves with the model of spherical particles including the particle-support interference effect (b).

cycling is obvious from the log-normal Pt nanoparticle size distributions shown in Figure $6 \mathrm{~b}$. The narrow initial distribution at an average diameter of $\bar{d}_{0}=2.2 \mathrm{~nm}$ collapses after the first high-potential cycle and continues to get broader and to shift to larger diameters to reach an average diameter of $\bar{d}_{500}=4.4 \mathrm{~nm}$ after 500 degradation cycles. Since the $\mathrm{IrO}_{2}-\mathrm{TiO}_{2}$ oxide is morphologically stable, cf. Figure 5, support corrosion appears to be not involved the observed Pt nanoparticle growth, which therefore can be seen as a result of electrochemical Ostwald ripening ${ }^{3}$ that proceeds via preferential dissolution of smaller Pt nanoparticles at high potentials and a subsequent redeposition of dissolved Pt ions on larger Pt nanoparticles at low potentials.

From the fit of Pt ASAXS results the relative evolution of the total Pt surface area can be determined. This is compared with the evolution of the Pt ECSA determined from glass cell experiments in Figure 7a. A drastic difference in the loss of both surface areas is established which can be explained as follows: Pt ECSA values at inital stages of degradation experiments can strongly underestimate the true $\mathrm{Pt}$ surface area as a result of surface blocking by adsorbed synthesis residuals. Such adsorbates can be oxidized and removed at high potentials leading to a Pt surface cleaning effect during the first high- potential degradation cycles that can be superimposed to the physical degradation of Pt surface area, which can be determined by ASAXS from the beginning. The superposition of cleaning and degradation can lead to a slower loss of the apparent ECSA in comparison to the surface area from ASAXS. Further confirmation of this interpretation can be drawn from Figure $7 b$ presenting the evolution of absolute values of mass-specific Pt surface area determined from ECSA (glass cell) and from ASAXS (in situ cell), respectively. The surface area is normalized with respect to the initial Pt mass at all stages of the degradation. From ASAXS, the initial mass-specific surface area is $\approx 130 \mathrm{~m}^{2} / \mathrm{g}_{\mathrm{Pt}}$, whereas initial ECSA values strongly underestimate the surface area $\left(\approx 55 \mathrm{~m}^{2} / \mathrm{g}_{\mathrm{Pt}}\right)$. In the course of degradation cycling both specific surface area values converge as a result of continuous cleaning of the Pt surface.

Also shown in Figure $7 \mathrm{a}$ is the relative evolution of the total $\mathrm{Pt}$ mass determined from the fits of Pt ASAXS results. Interestingly, approximately $84 \%$ of the initial Pt mass is preserved during the degradation protocol, which shows that $\mathrm{Pt}$ loss due to dissolution into the bulk electrolyte is negligible compared to the loss of Pt surface area resulting from electrochemical Ostwald ripening.
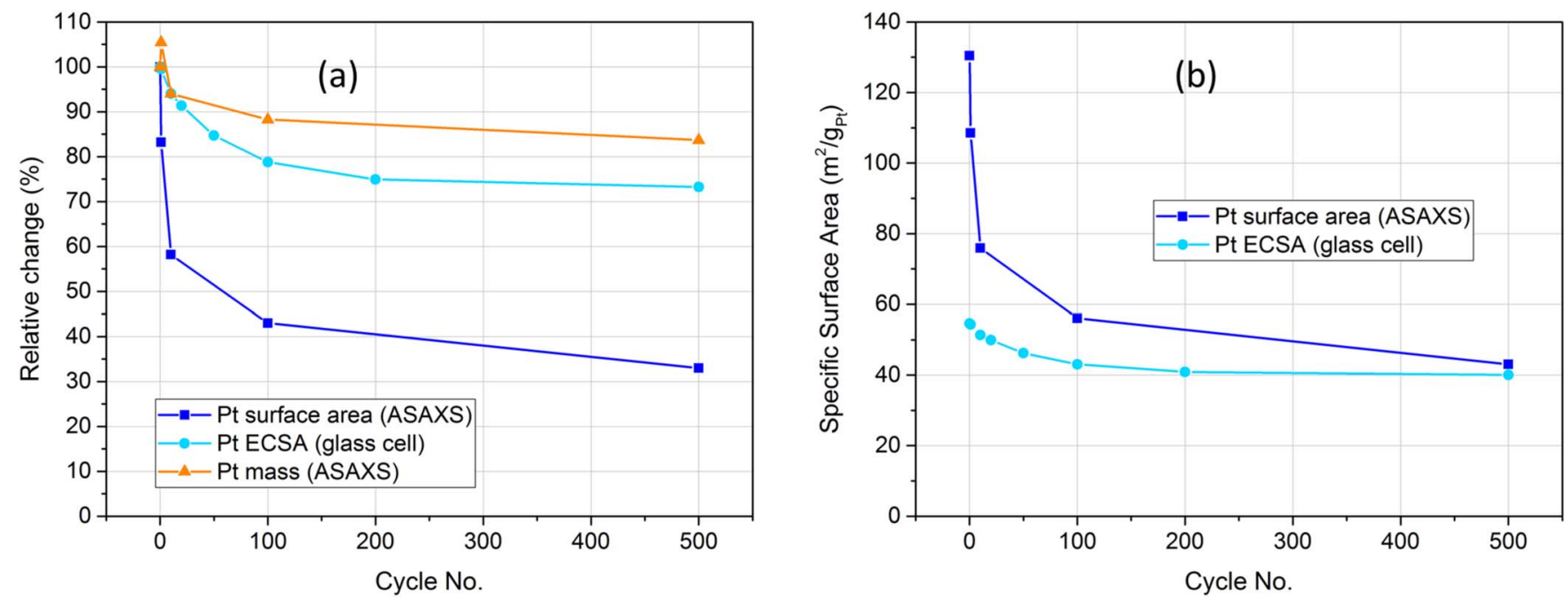

Figure 7. (a) Relative evolution of Pt surface area determined from ASAXS, Pt ECSA determined in a glass cell experiment, and total Pt mass determined from ASAXS as a function of the number of high-potential degradation cycles. (b) Evolution of specific Pt surface area w.r.t. the initial Pt mass as a function of the number of degradation cycles, once determined from ASAXS (in situ cell) and once from ECSA (glass cell), respectively. 


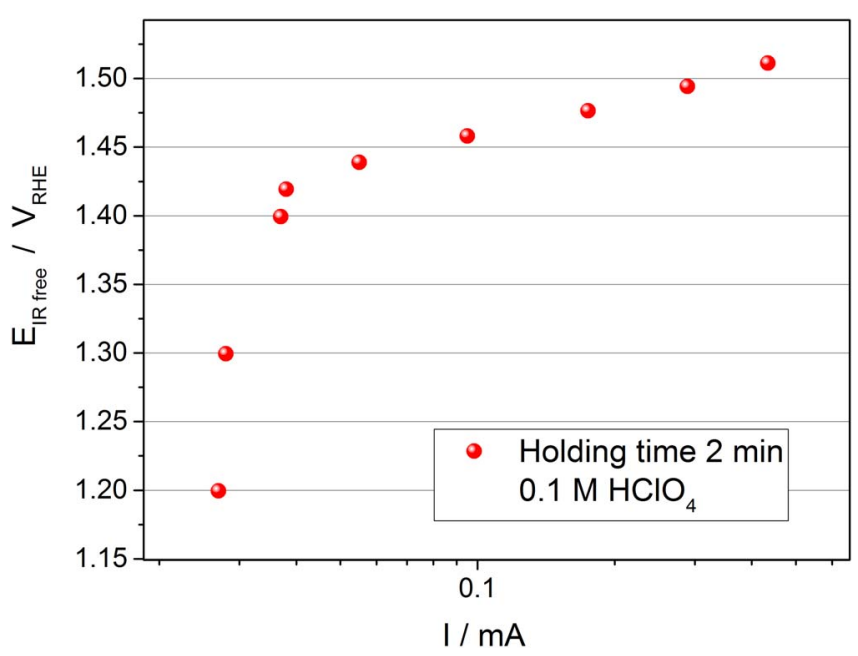

Figure 8. Tafel plot of the electrochemical data collected during in situ XAS at the Ir- $\mathrm{L}_{\mathrm{III}}$ edge for the $\mathrm{IrO}_{2}-\mathrm{TiO}_{2}$ OER catalyst.

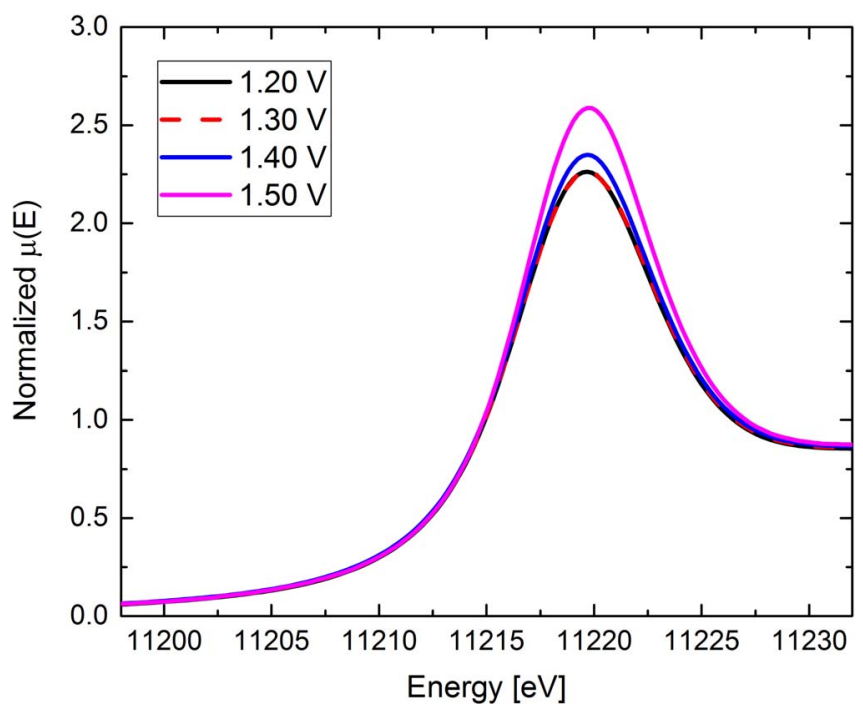

Figure 9. Normalized XANES spectra at the Ir- $\mathrm{L}_{\text {III }}$ edge for the $\mathrm{IrO}_{2}-\mathrm{TiO}_{2}$ OER catalyst at different applied potentials below and above OER onset.
In situ $\mathrm{XAS}$ results: Electronic state of $\mathrm{IrO}_{2}-\mathrm{TiO}_{2} \mathrm{OER}$ catalyst.-Figure 8 presents a Tafel plot of electrochemical currents for increasing potentials into the OER range recorded for the $\mathrm{IrO}_{2}$ $\mathrm{TiO}_{2}$ catalyst during in situ XAS experiments. Onset of oxygen evolution occurs around 1.4 $\mathrm{V}_{\mathrm{RHE}}$. Figure 9 presents the normalized XANES spectra at the Ir- $\mathrm{L}_{\mathrm{III}}$ edge collected at the respective potentials. The dominant "white line" peak remains constant for the potentials 1.2 $\mathrm{V}_{\mathrm{RHE}}$ and $1.3 \mathrm{~V}_{\mathrm{RHE}}$ below the OER onset. However, with OER onset at $1.4 \mathrm{~V}_{\mathrm{RHE}}$, the white line intensity increases and it continues to grow with further increasing potentials. The intensity of the white line peak is generally interpreted as a measure of the number of vacant Ir dstates and thus of the Ir partial oxidation state (charge density around Ir centers). ${ }^{30-32}$ Therefore, an increase of the white line peak intensity is an indication of the removal of electronic charge from the Ir sites with increasing potentials starting at the onset of OER.

Fast changes of the Ir partial oxidation state as a function of potential are well-known for electrochemically formed iridium oxide films ${ }^{30}$ and for sputtered iridium oxide films ${ }^{31}$ in the potential range below OER onset and are generally interpreted in terms of proton insertion/extraction into/from the bulk of the iridium oxide film. However, this proton intercalation process is suppressed for thermally prepared crystalline iridium oxides as used in our study, which is in good agreement with our observation of a constant electron density at Ir centers at potentials below OER onset. Hypothetically, the increase in Ir partial oxidation state in correlation with the onset of OER could be indirectly interpreted in terms of a transformation of the iridium oxide from its initial crystalline rutile structure into a more amorphous structure similar to the one of electrochemically formed iridium oxides. Such a structural change could allow an increase of the iridium valency beyond 4+, which was previously reported for amorphous iridium oxide layers formed electrochemically on metallic Ir electrodes. ${ }^{11}$ An intimate correlation between structural changes of the metal oxide and the onset of OER has been explained recently by the direct evolution of lattice oxygen (LOER) that is thermodynamically favoured under OER conditions. ${ }^{12}$

Presented in Figure 10 are iridium EXAFS spectra for the $\mathrm{IrO}_{2}$ $\mathrm{TiO}_{2}$ catalyst in $k$-space and in $R$-space which were recorded in situ within 1 min acquisition time at an electrode potential of $1.3 \mathrm{~V}_{\mathrm{RHE}}$. The quality of the EXAFS data is reflected in Figure 10a, which shows that reliable data could be collected up to $k \approx 12 \AA^{-1}$. The EXAFS data were then Fourier transformed into $R$-space and modeling of the spectra was performed to gain more insight regarding the local coordination environment of iridium. A simple two-shell model was used to fit the $\mathrm{IrO}_{2}-\mathrm{TiO}_{2}$ data based on the rutile structure of $\mathrm{IrO}_{2}$, which captures the key features of the local iridium environment up
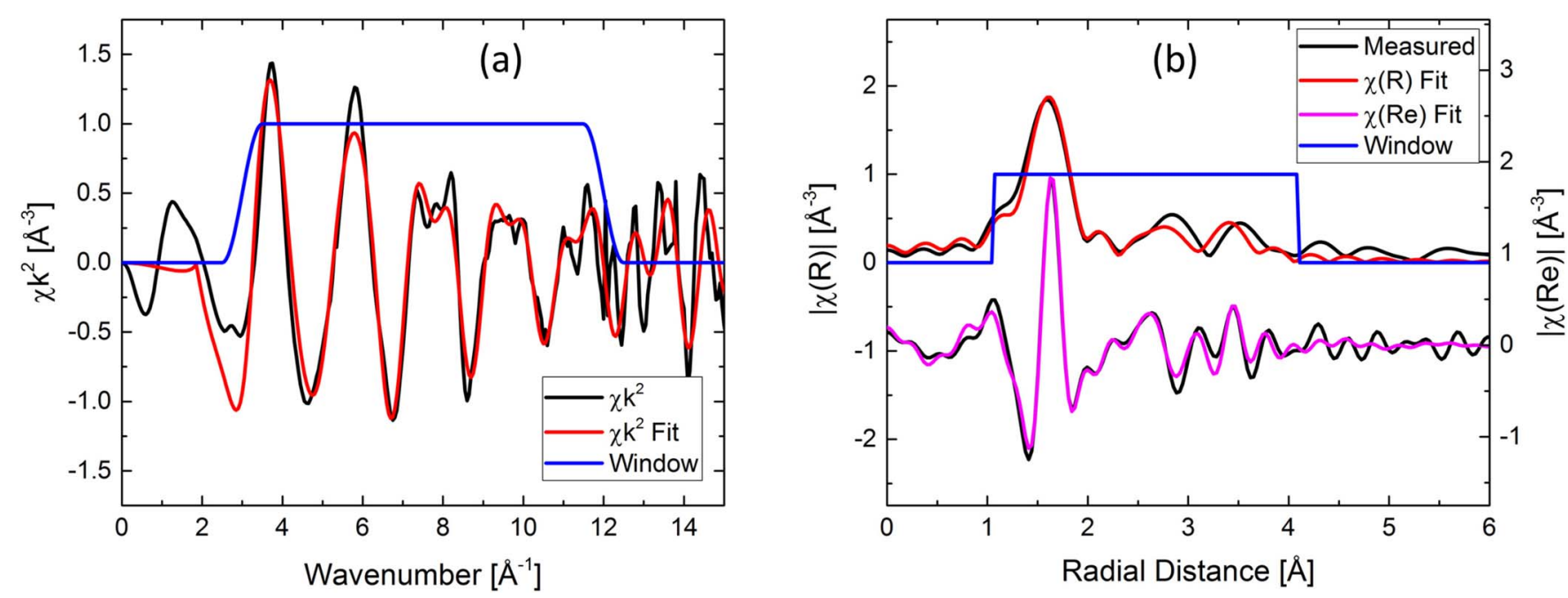

Figure 10. In situ iridium EXAFS spectra at a potential of $1.3 \mathrm{~V}_{\mathrm{RHE}}$ shown for the $\mathrm{IrO}_{2}-\mathrm{TiO}_{2}$ OER catalyst in $k$-space (a) and in $R$-space (b), respectively. Data was collected in situ within 1 min acquisition time. 


\begin{abstract}
Table I. Summary of the in situ EXAFS best fit parameters for $\mathrm{IrO}_{2}-\mathrm{TiO}_{2}$ at $1.3 \mathrm{~V}_{\mathrm{RHE}} . N$ denotes the coordination number of the given scattering path, $d$ indicates the refined path length, $\sigma^{2}$ represents the Debye-Waller factor, and $\Delta E_{0}$ is the energy shift. An amplitude reduction factor of $S_{0}^{2}=0.77$ was used for the fit. $k$ was kept within the range of $k=3-12 \AA^{-1}$.
\end{abstract}

\begin{tabular}{cccccc} 
Path & $N$ & $d / \AA$ & $\sigma^{2} / \AA^{2}$ & $\Delta E_{0} / \mathrm{eV}$ & $R$-factor \\
\hline O1.2 & $4.9 \pm 0.6$ & $1.988 \pm 0.011$ & $0.0017 \pm 0.0016$ & & \\
Ir1.1 & $8.9 \pm 7.2$ & $3.148 \pm 0.011$ & $0.0138 \pm 0.0104$ & $13.00 \pm 1.18$ & 0.0371 \\
Ir1.2 & $2.0 \pm 3.7$ & $3.545 \pm 0.011$ & $0.0010 \pm 0.0096$ & &
\end{tabular}

to ca. $4 \AA$. The six nearest neighboring oxygen atoms forming the first coordination shell were described using a single $\mathrm{Ir}-\mathrm{O}$ scattering path $\left(R_{\text {eff }}=1.999 \AA\right)$. The iridium atoms that comprise the second shell were then described using two separate $\mathrm{Ir}-\mathrm{Ir}$ scattering paths ( $R_{\text {eff }}=3.159$ and $3.556 \AA$, respectively). It should be noted that the fitting of the first Ir-O shell remains relatively unchanged with the addition of the Ir-Ir scattering paths. For simplicity, the fit excludes the longer $\mathrm{Ir}-\mathrm{O}$ scattering paths $(>3.0 \AA)$ and multiple scattering paths since a reasonable fit could be achieved in their absence (as is evidenced by the $\chi(\mathrm{Re})$ best fit).

Fitting of the first peak within the range of ca. 1-2 $\AA$, which mainly represents the 6 nearest neighboring oxygen atoms forming the distorted octahedron, yields an average Ir-O bond length of $1.988 \AA$. This agrees well with the average $\mathrm{Ir}-\mathrm{O}$ bond length of $1.98 \AA$ reported for $\mathrm{IrO}_{2} \cdot{ }^{33}$ The details and results of the fit are further summarized in Table I.

\section{Conclusions}

An electrochemical three-electrode flow-cell for in situ SAXS (transmission mode) and XAS (transmission and fluorescence mode) studies at synchrotron X-ray sources was developed. The flow of electrolyte guarantees constant experimental conditions, preventing X-ray induced electrolyte degradation and heating, and carrying-out bubbles of gases that are evolved on the electrodes. Thus, in situ studies under minor gas evolution conditions, e.g. on OER catalyst materials, are possible. A special arrangement of the three electrodes enables electrochemical measurements with low residual ohmic resistance $\approx 10 \Omega$. The cell was designed for the study of technically relevant high surface area catalyst materials in porous electrode layers that were prepared by spray-coating on conductive Kapton substrates.

The flow-cell was applied in an in situ anomalous SAXS study of PEFC-relevant degradation properties of a $\mathrm{Pt} / \mathrm{IrO}_{2}-\mathrm{TiO}_{2}$ ORR catalyst in an electrochemical protocol with potential cycles up to $1.5 \mathrm{~V}_{\mathrm{RHE}}$ that simulates the corrosive conditions at PEFC cathodes during start and stop events. Electrochemical Ostwald ripening was found as the dominant loss mechanism for the Pt surface area. Pt mass loss due to dissolution into the electrolyte flow only marginally contributed to the overall degradation.

In another experiment, the flow-cell was used in an in situ XAS study of the electronic properties of Ir centers inside an $\mathrm{IrO}_{2}-\mathrm{TiO}_{2}$ catalyst under OER conditions. XANES analysis revealed a constant electronic state of Ir for potentials below OER onset, and a direct correlation between the start of a continuous increase of Ir partial oxidation state and the onset of oxygen evolution. The quality of in situ-acquired XAS data was confirmed by the corresponding iridium EXAFS spectra that were fitted with a model based on the rutile structure of $\mathrm{IrO}_{2}$.

\section{Acknowledgments}

This work was supported by CCEM Switzerland and Umicore AG \& Co KG within the project DuraCat. The authors thank the Swiss
Light Source (SLS, Paul Scherrer Institut, Villigen, Switzerland) for providing synchrotron radiation beamtime at the cSAXS beamline and at the SuperXAS beamline. M. Horisberger is gratefully acknowledged for help in connection with gold sputtering and Dr. A. Diaz for assistance during cSAXS beamtime. EF gratefully acknowledges the Ambizione Program of the Swiss National Science Foundation. R. Mohamed and the Electron Microscope Unit at the University of Cape Town, South Africa, are acknowledged for providing the TEM image of a Pt/C catalyst. We gratefully thank Dr. M. Dadras from CSEM, Switzerland, for the TEM image of the $\mathrm{Pt} / \mathrm{IrO}_{2}-\mathrm{TiO}_{2}$ catalyst.

\section{References}

1. C. A. Reiser, L. Bregoli, T. W. Patterson, J. S. Yi, J. D. L. Yang, M. L. Perry, and T. D. Jarvi, Electrochem. Solid-State Lett., 8, A273 (2005)

2. H. Schulenburg, B. Schwanitz, N. Linse, G. G. Scherer, A. Wokaun, J. Krbanjevic, R. Grothausmann, and I. Manke, J. Phys. Chem. C, 115, 14236 (2011).

3. Y. Shao-Horn, W. C. Sheng, S. Chen, P. J. Ferreira, E. F. Holby, and D. Morgan, Top. Catal., 46, 285 (2007)

4. J. C. Meier, C. Galeano, I. Katsounaros, A. A. Topalov, A. Kostka, F. Schüth, and K. J. J. Mayrhofer, ACS Catal., 2, 832 (2012).

5. K. J. J. Mayrhofer, J. C. Meier, S. J. Ashton, G. K. H. Wiberg, F. Kraus, M. Hanzlik, and M. Arenz, Electrochem. Commun., 10, 1144 (2008).

6. M. J. Williamson, R. M. Tromp, P. M. Vereecken, R. Hull, and F. M. Ross, Nat. Mater, 2, 532 (2003)

7. H. G. Haubold, X. H. Wang, H. Jungbluth, G. Goerigk, and W. Schilling, J. Mol. Struct., 383, 283 (1996).

8. H. B. Stuhrmann, Adv. Polym. Sci., 67, 123 (1985).

9. T. Binninger, M. Garganourakis, J. Han, A. Patru, E. Fabbri, O. Sereda, R. Kötz, A. Menzel, and T. J. Schmidt, Phys. Rev. Applied, 3, 024012 (2015).

10. K. J. May, C. E. Carlton, K. A. Stoerzinger, M. Risch, J. Suntivich, Y.-L. Lee, A. Grimaud, and Y. Shao-Horn, J. Phys. Chem. Lett., 3, 3264 (2012).

11. N. Danilovic, R. Subbaraman, K.-C. Chang, S. H. Chang, Y. J. Kang, J. Snyder, A. P. Paulikas, D. Strmcnik, Y.-T. Kim, D. Myers, V. R. Stamenkovic, and N. M. Markovic, J. Phys. Chem. Lett., 5, 2474 (2014).

12. T. Binninger, R. Mohamed, K. Waltar, E. Fabbri, P. Levecque, R. Kötz, and T. J. Schmidt, Sci. Rep., 5, 12167 (2015).

13. A. Bergmann, E. Martinez-Moreno, D. Teschner, P. Chernev, M. Gliech, J. F. de Araujo, T. Reier, H. Dau, and P. Strasser, Nat. Commun., 6, 8625 (2015).

14. S. Mukerjee, S. Srinivasan, M. P. Soriaga, and J. McBreen, J. Electrochem. Soc., 142, 1409 (1995).

15. C. Roth, N. Martz, T. Buhrmester, J. Scherer, and H. Fuess, Phys. Chem. Chem. Phys., 4, 3555 (2002).

16. R. J. K. Wiltshire, C. R. King, A. Rose, P. P. Wells, M. P. Hogarth, D. Thompsett, and A. E. Russell, Electrochim. Acta, 50, 5208 (2005)

17. O. Petrova, C. Kulp, M. W. van den Berg, K. V. Klementiev, B. Otto, H. Otto, M. Lopez, M. Bron, and W. Grünert, Rev. Sci. Instrum., 82, 044101 (2011).

18. J. Roth, J. Eller, and F. N. Büchi, J. Electrochem. Soc., 159, F449 (2012).

19. M. E. Herron, S. E. Doyle, S. Pizzini, K. J. Roberts, J. Robinson, G. Hards, and F. C. Walsh, J. Electroanal. Chem., 324, 243 (1992)

20. X. Tuaev and P. Strasser In Polymer Electrolyte Membrane and Direct Methanol Fuel Cell Technology; C. Hartnig and C. Roth, Eds.; Woodhead Publishing, 2012; Vol. 2; pp 87.

21. J. Tillier, T. Binninger, M. Garganourakis, A. Patru, E. Fabbri, T. J. Schmidt, and O. Sereda, J. Electrochem. Soc., 163, H913 (2016).

22. J. P. Suchsland, B. Klose-Schubert, D. Herein, T. Martin, C. Eickes, and M. Lennartz, ECS Trans., 50, 1659 (2013).

23. A. Patru, A. Rabis, S. E. Temmel, R. Kötz, and T. J. Schmidt, Catal. Today, 262, 161 (2016).

24. B. Henrich, A. Bergamaschi, C. Broennimann, R. Dinapoli, E. F. Eikenberry, I. Johnson, M. Kobas, P. Kraft, A. Mozzanica, and B. Schmitt, Nucl. Instrum. Methods Phys. Res., Sect. A, 607, 247 (2009).

25. F. Zhang, J. Ilavsky, G. G. Long, J. P. G. Quintana, A. J. Allen, and P. R. Jemian, Metall. Mater. Trans. A, 41, 1151 (2010).

26. T. Binninger, E. Fabbri, R. Kötz, and T. J. Schmidt, ECS Trans., 58, 1835 (2013).

27. T. Binninger, E. Fabbri, R. Kötz, and T. J. Schmidt, J. Electrochem. Soc., 161, H121 (2013).

28. O. Müller, M. Nachtegaal, J. Just, D. Lützenkirchen-Hecht, and R. Frahm, J. Synchrotron Radiat, 23, 260 (2016).

29. B. Ravel and M. Newville, J. Synchrotron Radiat., 12, 537 (2005).

30. A. R. Hillman, M. A. Skopek, and S. J. Gurman, Phys. Chem. Chem. Phys., 13, 5252 (2011).

31. T. Pauporté, D. Aberdam, J.-L. Hazemann, R. Faure, and R. Durand, J. Electroanal. Chem., 465, 88 (1999).

32. A. M. Cruz, L. Abad, N. M. Carretero, J. Moral-Vico, J. Fraxedas, P. Lozano, G. Subías, V. Padial, M. Carballo, J. E. Collazos-Castro, and N. Casañ-Pastor, J. Phys. Chem. C, 116, 5155 (2012).

33. V. Pfeifer, T. E. Jones, J. J. Velasco Velez, C. Massue, M. T. Greiner, R. Arrigo, D. Teschner, F. Girgsdies, M. Scherzer, J. Allan, M. Hashagen, G. Weinberg, S. Piccinin, M. Hävecker, A. Knop-Gericke, and R. Schlögl, Phys. Chem. Chem. Phys., 18, 2292 (2016) 